\title{
Health Psychology
}

\section{Associations Between Coping Strategies and Mental Health in Individuals With Type 2 Diabetes: Prospective Analyses}

Rachel J. Burns, Sonya S. Deschênes, and Norbert Schmitz

Online First Publication, July 20, 2015. http://dx.doi.org/10.1037/hea0000250

CITATION

Burns, R. J., Deschênes, S. S., \& Schmitz, N. (2015, July 20). Associations Between Coping Strategies and Mental Health in Individuals With Type 2 Diabetes: Prospective Analyses. Health Psychology. Advance online publication. http://dx.doi.org/10.1037/hea0000250 


\title{
Associations Between Coping Strategies and Mental Health in Individuals With Type 2 Diabetes: Prospective Analyses
}

\author{
Rachel J. Burns, Sonya S. Deschênes, and Norbert Schmitz \\ McGill University and Douglas Mental Health University Institute, Montreal, Quebec, Canada
}

\begin{abstract}
Objective: Individuals with type 2 diabetes are at greater risk of developing a number of mental health conditions, including depression, anxiety, and diabetes-related distress, than individuals without type 2 diabetes. Cross-sectional studies suggest that some coping strategies may increase the risk of mental health conditions in individuals with diabetes, whereas others may be protective. This study extends the cross-sectional evidence base with a prospective study. Methods: Data were collected annually for 2 years from a community sample of 1,742 adults with type 2 diabetes. Coping strategies were measured at baseline and mental health conditions were assessed at each time point with self-report symptom measures. For comparison, cross-sectional and prospective analyses were conducted. Results: Crosssectional analyses demonstrated that task-oriented coping was negatively associated with the likelihood of each of the mental health conditions, emotion-oriented coping was positively associated with the likelihood of each condition, and avoidance-oriented coping showed no association. Prospective analyses revealed that among individuals who did not have elevated depressive symptoms at baseline, only emotion-oriented coping predicted the likelihood of developing major depression syndrome during follow-up. Similar patterns of results were observed for elevated anxiety symptoms and diabetes-related distress. Conclusions: Cross-sectional results differed from prospective results. Only emotion-oriented coping appears to play a role in the development of depressive symptoms, anxiety symptoms, and diabetes-related distress. Results underscore the importance of examining prospective associations and suggest that interventions targeting specific coping strategies might alleviate mental health problems in individuals with type 2 diabetes.
\end{abstract}

Keywords: coping, diabetes, depression, anxiety, diabetes-related distress

Supplemental materials: http://dx.doi.org/10.1037/hea0000250.supp

Type 2 diabetes is associated with an increased risk of several mental health conditions, including depression (Mezuk, Eaton, Albrecht, \& Golden, 2008), anxiety (Smith, Beland, et al., 2013), and diabetes-related distress (Fisher et al., 2008). In addition to being associated with poor quality of life and physical functioning (Hendriks et al., 2014; Lecrubier, 2001), these mental health conditions are associated with factors that may intensify diabetes severity, such as poor glycemic control and decreased adherence to medical and behavioral regimens (de Groot, Anderson, Freedland, Clouse, \& Lustman, 2001). Identifying risk and protective factors-particularly those that are modifiable-that affect the likelihood of developing these mental health conditions among indi-

Rachel J. Burns and Sonya S. Deschênes, Department of Psychiatry, McGill University and Douglas Mental Health University Institute, Montreal, Quebec, Canada; Norbert Schmitz, Departments of Psychiatry; Epidemiology and Biostatistics, McGill University and Douglas Mental Health University Institute, Montreal, Quebec, Canada.

The EDIT study is funded by Canadian Institutes of Health Research Grant MOP-106514.

Correspondence concerning this article should be addressed to Dr. Rachel J. Burns, Douglas Mental Health University Institute, 6875 Boul. Lasalle, Frank B. Common Pavilion, Montreal QC H4H 1R3. E-mail: Rachel.burns@mail.mcgill.ca viduals with type 2 diabetes may help to minimize their deleterious effects by providing an evidence base for interventions. The purpose of this paper is to explore the prospective associations between coping strategies and the development of depressive symptoms, anxiety symptoms, and diabetes-related distress.

The literature suggests that chronic diseases, such as type 2 diabetes, are an ongoing source of stress that must be managed to protect one's mental health (Stanton, Revenson, \& Tennen, 2007). Coping strategies are the thoughts and behaviors used to manage the emotional and logistical demands of situations that are appraised as stressful (Folkman \& Moskowitz, 2004). The central role that coping plays in the progressive relationship between stress and poor mental health outcomes has been theoretically and empirically established (Lazarus, 1993). Classic coping theory distinguishes between two types of coping strategies: emotionoriented and task-oriented (Folkman \& Lazarus, 1980). Emotionoriented coping involves efforts to regulate negative emotions that emerge when confronted with a stressor, whereas task-oriented coping involves active steps to modify a stressor or one's association to it (Folkman \& Lazarus, 1980). Avoidance-oriented coping has emerged as a third class of coping strategies and involves efforts to avoid coming into contact with or thinking about a stressor (Endler \& Parker, 1990). Though individuals tend to have habitual or preferred coping strategies (Moos \& Holahan, 2003), coping strategies are modifiable (Kroese, Adriaanse, Vinkers, van de Schoot, \& de Ridder, 2014; D’Eramo Melkus et al., 2010). 
Cross-sectional studies have examined the associations between coping strategies and depression and anxiety in people with diabetes. Several studies with samples comprised of individuals with type 1 diabetes or a combination of individuals with type 1 and type 2 diabetes report negative associations between task-oriented coping and depression and anxiety symptoms, and positive associations between emotion-oriented coping and depression and anxiety symptoms (Karlsen, Idsoe, Dirdal, Rokne Hanestad, \& Bru, 2004; Smári \& Valtýsdóttir, 1997; Tuncay, Musabak, Gok, \& Kutlu, 2008). However, findings in this area are mixed; negative associations between emotion-oriented coping and depression and anxiety in individuals with diabetes have also been reported (see Duangdao \& Roesch, 2008 for review). These mixed findings may be attributable to differences in the measurement of emotionoriented and task-oriented coping across studies. For example, some measures of emotion-oriented coping assess how often strategies that seek to minimize or alleviate negative affect are employed (e.g., seeking social support; Moos \& Moos, 1988), whereas others assess the extent to which one experiences negative affect when encountering a stressor (e.g., feeling nervous about not being able to cope; Endler \& Parker, 1990). Few studies have examined avoidance-oriented coping; a single cross-sectional study found no associations between avoidance-oriented coping and depression or anxiety symptoms among individuals with type 1 diabetes (Smári \& Valtýsdóttir, 1997).

In general, research in this area has been limited to crosssectional data, so temporal precedence cannot be established (Duangdao \& Roesch, 2008). Therefore, it is unclear whether (a) coping strategies affect mental health, (b) mental health affects coping strategies, and/or (c) a third unmeasured variable affects both coping strategies and mental health in individuals with diabetes. Prospective studies can offer clarity by examining how coping strategies are associated with the development of mental health conditions in people who do not have these conditions at baseline.

Furthermore, the majority of research in this area has focused on individuals with type 1 diabetes or has not distinguished between individuals with type 1 and type 2 diabetes (Duangdao \& Roesch, 2008; exceptions Macrodimitris \& Endler, 2001; Shah, Gupchup, Borrego, Raisch, \& Knapp, 2012). type 2 diabetes is distinguished from type 1 diabetes in its etiology; the onset of type 2 diabetes can typically be prevented or delayed with a healthy lifestyle, whereas type 1 diabetes cannot be prevented (Alberti \& Zimmet, 1998). The developmental period during which the disease emerges also differs; type 2 diabetes usually emerges during adulthood, whereas type 1 diabetes typically emerges during childhood or adolescence (Alberti \& Zimmet, 1998). Given these differences, it is not surprising that the prevalence of specific coping strategies differs between individuals with type 1 and type 2 diabetes (Tuncay et al., 2008). Accordingly, the associations between coping strategies and mental health outcomes observed in individuals with type 1 diabetes might not generalize to those with type 2 diabetes.

Previous studies of coping strategies and mental health in individuals with diabetes have generally been limited to depression and anxiety. However, diabetes-related distress is also an important mental health consideration (Fisher et al., 2008). Diabetesrelated distress is a construct that captures the set of worries, concerns, and fears that are specific to diabetes and its management (e.g., emotional burden, potential for complications and loss of functioning, concern about quality of medical care; Fisher, Gonzalez, \& Polonsky, 2014). It is distinguished from depression and anxiety because it encompasses a broader set of affective experiences that are elicited in specific response to diabetes (Fisher et al., 2014; Gonzalez, Fisher, \& Polonsky, 2011). High levels of diabetes-related distress are associated with poor quality of life, glycemic control, and self-care (Fisher et al., 2009). A single cross-sectional study of adults with type 2 diabetes indicated that diabetes-related distress was associated with the use of self-blame and behavioral disengagement strategies, which may be interpreted as forms of emotion-oriented and task-oriented coping, respectively (Karlsen, Oftedal, \& Bru, 2012). However, the measure of distress used in this study has been criticized for not adequately capturing all aspects of diabetes-related distress (e.g., physicianrelated distress; Polonsky et al., 2005.

The present study seeks to expand upon the extant literature by examining prospective associations between coping strategies and mental health outcomes in a community sample of individuals with type 2 diabetes. To increase the clinical utility of the present study, symptom assessments are based on meaningful cut-off scores for established measures of depressive symptoms, anxiety symptoms, and diabetes-related distress. For the sake of comparison, crosssectional associations between task-, emotion-, and avoidanceoriented coping and each of the three mental health outcomes were first calculated. Then, coping strategies at baseline were used to predict the likelihood of developing each of the three mental health conditions during a 2-year follow-up among individuals who did not have these conditions at baseline.

\section{Method}

\section{Data Source}

Data were from the first three waves of the Evaluation of Diabetes Treatment (EDIT) study, which is an ongoing, longitudinal, telephone survey study of community-dwelling, Englishand French-speaking residents of the Canadian province of Québec (Smith, Gariepy, et al., 2013). The primary objective of EDIT is to study the transition from oral hypoglycemic mediation to insulin. Participants were recruited by random digit dialing and mailing letters to individuals with a diagnosis of type 2 diabetes registered in the Québec health insurance system, the Régie de l'assurance maladies du Québec (RAMQ). Eligibility criteria for participation in EDIT at baseline were: self-report of having been diagnosed by a doctor with type 2 diabetes within the last 10 years, being insulin-naïve, and being between 40 and 75 years of age. Baseline data collection occurred in 2011 and data are collected annually. The Research Ethics Committee of the Douglas Mental Health University Institute, an affiliate of McGill University, approved the study protocol. Participants were included in the present analyses if they had complete data on the measures included in the regression models, which are described subsequently.

\section{Participants}

At baseline ( $n=2,028)$, the sample was predominantly Caucasian $(93 \%)$ and married/living as married $(65 \%)$. Men and women were nearly equally represented ( $51 \%$ men) and the mean age was 60.51 years $(S D=8.37)$. Thirty-nine percent of participants re- 
ported working full- or part-time; the remainder were retired $(46.1 \%)$ or not working (14.9\%). On average, participants had been diagnosed with type 2 diabetes for 4.59 years $(S D=3.10)$. The majority of participants were overweight $(38.2 \%)$ or obese $(44.4 \%)$. The sample is further detailed elsewhere (Smith \& Schmitz, 2014).

During baseline data collection, participants were asked if they would like to participate in the study during follow-up. Only participants who indicated that they might like to participate during follow-up were subsequently contacted $(n=1,691)$. Six participants were reported by family members to be deceased by follow-up 2. At follow-up 1, participation was declined by 293 of the participants who were reached. Of the participants who were reached at follow-up 2, 221 declined to participate. The remaining participants lost to follow-up could not be reached or located.

Of the 2,028 participants included in the EDIT study at baseline, 286 were excluded from the cross-sectional analyses for having missing data on at least one of the measures included in the models $(n=1,742)$. Coping strategies, age, diabetes duration, and sex did not significantly differ between those included and excluded (all $p>$.05). Those who were included had significantly fewer diabetes complications $\left(M_{\text {excluded }}=1.46 ; M_{\text {included }}=1.08, t(1907)=\right.$ 4.01, $p<.001)$. Sample sizes for the prospective analyses are reported in Table 2 .

\section{Measures}

Coping strategies. Coping strategies were measured at baseline with the 21-item version of the Coping Inventory for Stressful Situations (CISS-21; Endler \& Parker, 1994). CISS-21 is a selfreport measure of general coping strategies. It assesses how frequently respondents engage in task-oriented (e.g., determine a course of action and follow it; 7 items; $\alpha=.85$ ), emotion-oriented (e.g., feel anxious about not being able to cope; 7 items; $\alpha=.83$ ), and avoidance-oriented coping (e.g., buy myself something; 7 items; $\alpha=.75$ ) when encountering a difficult, stressful, or upsetting situation. Responses are made on a 5-point scale $(1=$ not at all; 5 = very much) and aggregated.

Depression. Depression was assessed at all time points with the Patient Health Questionnaire (PHQ-9; Kroenke, Spitzer, \& Williams, 2001), which was developed as a screening tool for major depressive disorder in primary care settings. The PHQ-9 is a 9-item self-report measure of symptoms of depression (e.g., feeling down, depressed, or hopeless) experienced within the last 2 weeks $(\alpha=.79, .82$, and .80 at baseline, follow-up 1 , and follow-up 2, respectively). Items are rated on a scale ranging from 0 (not at all) to 3 (nearly every day). Though not a clinical assessment, the PHQ-9 identifies cases of major and minor depression syndrome by accounting for the diagnostic criteria for depression, rather than relying on summed symptom scores. To meet the criteria of major depression syndrome, respondents must endorse either dysphoria or anhedonia, and at least four of the remaining items on more than half the days in the past two weeks (score $\geq 2$ ). This measure has moderate concordance with clinical diagnosis of major depressive disorder (Eaton, Hall, Macdonald, \& McKibben, 2007). Not meeting the criteria for major depression syndrome was coded as 0 and meeting the criteria for major depression syndrome was coded as 1 . In prospective analyses, meeting the criteria for major depression syndrome at one or both follow-up points was coded as 1. The PHQ-9 also assesses minor depression syndrome. To meet the criteria of minor depression syndrome, respondents must endorse either dysphoria or anhedonia, and two or three other items as occurring on more than half the days in the past 2 weeks. For both minor and major depression syndrome, the item measuring suicidal ideation was counted if it was scored at 1 (several days) or higher.

Anxiety. Anxiety was measured with the Generalized Anxiety Disorder-7 item measure at all time points (GAD-7;(Spitzer, Kroenke, Williams, \& Lowe, 2006). The GAD-7 is a self-report measure of symptoms of generalized anxiety (e.g., feeling nervous, anxious, or on edge) experienced within the past two weeks. Items are rated on a scale ranging from 0 (not at all) to 3 (nearly every day). Total scores are summed $(\alpha=.85, .88$, and .87 at baseline, follow-up 1, and follow-up 2, respectively) and categorized as: no anxiety (score 0-4), mild anxiety (5-9), moderate anxiety (1014 ), or severe anxiety (15 or higher). A score of 10 is regarded as a clinically meaningful cut-point because it represents the best balance between sensitivity and specificity of generalized anxiety disorder (i.e., most patients with scores below 10 did not have generalized anxiety disorder, whereas most patients with scores of 10 or above did have generalized anxiety disorder; Spitzer et al., 2006). Low anxiety symptoms $(<10.0)$ were coded as 0 in the present analyses, and elevated anxiety symptoms $(\geq 10.0)$ were coded was 1 . In prospective analyses, scoring in the elevated anxiety symptoms range at one or both follow-up time points was coded as 1 .

Diabetes-related distress. Diabetes-related distress was measured at all time points with the Diabetes Distress Scale (Polonsky et al., 2005), which is a 17-item self-report scale that assesses emotional burden, physician-related distress, regimen-related distress, and interpersonal distress. Respondents rate the degree to which each potential source of distress has bothered or distressed them on a 6 -point scale $(1=$ not a problem; $6=$ a very serious problem). Item scores are aggregated to form a global score $(\alpha=$ $.93, .93$, and .94 at baseline, follow-up 1, and follow-up 2, respectively). Global scores can be categorized as: no to little distress (score $<2.0$ ), moderate distress $(2.0-2.9)$, or high distress $(\geq 3.0$; Fisher, Hessler, Polonsky, \& Mullan, 2012). Consistent with past literature, the moderate and high distress categories were collapsed and coded as 1 (Fisher et al., 2012). Respondents scoring in the no to little distress category were coded as 0 . In prospective analyses, scoring in the moderate or severe distress range at one or both follow-up time points was coded as 1 .

Covariates. Covariates were measured at baseline and included demographic and disease-related characteristics. Demographic characteristics can be proxies for social and material resources, which can influence both coping and mental health (Cole \& Dendukuri, 2003; Gåfvels \& Wändell, 2006; Lorant et al., 2007; Tuncay et al., 2008). Demographic covariates included age, sex, current marital status (married/living as married, widowed/ divorced/separated, never married), ethnicity (White/Caucasian, Black/African American, Aboriginal, Hispanic, South Asian, Asian, Other), and education (less than secondary school graduation, secondary school graduation, some postsecondary, postsecondary graduation). Poor physical health may influence one's mental health and coping resources (de Groot, et al., 2001; Fisher et al., 2008; Smári \& Valtýsdóttir, 1997). Disease-related covariates included number of physical comorbidities (range $0-8$ ), dia- 
betes duration in years, and diabetes complications. Diabetes complications were measured with the Diabetes Complications Index (Fincke et al., 2005), which is a 17-item self-report measure that assesses and sums complications including coronary artery disease, cerebrovascular disease, peripheral vascular disease, neuropathy, retinopathy, and foot problems. Nominal covariates were dummy coded in regression models.

\section{Analysis}

Cross-sectional analyses. To determine the cross-sectional associations between coping strategies and the likelihood of major depression syndrome, elevated anxiety symptoms, and moderate to severe diabetes-related distress at baseline, each of these conditions was entered as the outcome variable in separate binary logistic regression models. In each model, covariates were entered into the first step; task-oriented, emotion-oriented, and avoidanceoriented coping were entered into the second step.

Prospective analyses. Minor depression is associated with an increased risk of developing major depression (Horwath, Johnson, Klerman, \& Weissman, 1992; Wells, Burnam, Rogers, Hays, \& Camp, 1992). Therefore, to most rigorously examine the associations between coping strategies and the likelihood of developing major depression syndrome, only individuals with neither minor nor major depression syndrome at baseline were included in the prospective analysis predicting major depression syndrome. Similarly, only individuals scoring in the no anxiety range and in the no to little diabetes-related distress range at baseline were included in analyses predicting elevated anxiety symptoms and moderate to severe diabetes-related distress, respectively. Three separate bi- nary logistic regressions tested the prospective associations between coping strategies and the development of each of the mental health outcomes of interest. In all models, covariates were entered into the first step; task-oriented, emotion-oriented, and avoidanceoriented coping were entered into the second step.

\section{Results}

Correlations between coping strategies, mental health outcomes, and covariates are presented in Table 1. In general, coping strategies were highly correlated. Mean scores were 2.93, 2.05, and 2.51 for task-oriented $(S D=.91$, observed range 1.00-5.00), emotion-oriented $(S D=.76$, observed range 1.00-5.00), and avoidance-oriented coping $(S D=.75$, observed range 1.00-4.71), respectively.

At baseline, 93 participants met the criteria for major depression syndrome, 148 met the criteria for elevated anxiety symptoms, and 377 met the criteria for moderate or severe diabetes-related distress. Among the 1,044 participants who had neither major nor minor depression syndrome at baseline and completed at least one follow-up assessment, 45 (4.31\%) developed major depressive syndrome during follow-up. Of the 763 participants who scored in the no anxiety range at baseline and completed at least one follow-up assessment, 26 (3.41\%) developed elevated anxiety symptoms during follow-up. Finally, of the 951 participants who were classified as having no to little diabetes-related distress at baseline and completed at least one follow-up assessment, 150 (15.77\%) developed moderate or severe distress during follow-up.

Table 1

Descriptive Statistics and Bivariate Correlations Between Coping Strategies, Mental Health Outcomes, and Covariates

\begin{tabular}{|c|c|c|c|c|c|c|c|c|c|c|c|}
\hline Variable & 1 & 2 & 3 & 4 & 5 & 6 & 7 & 8 & 9 & $M(S D) / \%$ & Range \\
\hline 1. Task-oriented coping & & & & & & & & & & $2.93(.9)$ & $1-5$ \\
\hline 2. Emotion-oriented coping & $.45^{* *}$ & & & & & & & & & $2.05(.8)$ & $1-5$ \\
\hline 3. Avoidance-oriented coping & $.53^{* *}$ & $.41^{* *}$ & & & & & & & & $2.51(.8)$ & $1-4.71$ \\
\hline 4. Major depression syndrome (baseline) & $.05^{*}$ & $.35^{* *}$ & $.05^{*}$ & & & & & & & $5.9 \%$ & \\
\hline 5. Elevated anxiety symptoms (baseline) & $.07^{* * *}$ & $.42^{* * *}$ & $.08^{* * *}$ & $.53^{* *}$ & & & & & & $9.6 \%$ & \\
\hline 6. Moderate/severe diabetes distress (baseline) & $.11^{* *}$ & $.36^{* * *}$ & $.15^{* *}$ & $.25^{* *}$ & $.30^{* *}$ & & & & & $22.4 \%$ & \\
\hline 7. Major depression syndrome (follow-up) & .04 & $.27^{* * *}$ & .03 & $.34^{* *}$ & $.33^{* *}$ & $.19^{* * *}$ & & & & $5.4 \%$ & \\
\hline 8. Elevated anxiety symptoms (follow-up) & $.08^{* *}$ & $.34^{* *}$ & $.10^{* * *}$ & $.28^{* *}$ & $.43^{* *}$ & $.20^{* *}$ & $.48^{* *}$ & & & $7.9 \%$ & \\
\hline 9. Moderate/severe diabetes distress (follow-up) & $.09^{* * *}$ & $.34^{* * *}$ & $.11^{* * *}$ & $.25^{* *}$ & $.24^{* *}$ & $.43^{* *}$ & $.25^{* *}$ & $.22^{* * *}$ & & $20.8 \%$ & \\
\hline 10. Age & $-.17^{* *}$ & $-.13^{* *}$ & $-.07^{* * *}$ & -.03 & -.03 & $-.13^{* *}$ & $-.09^{* *}$ & -.01 & $-.15^{* *}$ & $60.51(8.4)$ & $40-75$ \\
\hline 11. Sex: male & $-.09^{* *}$ & $-.21^{* * *}$ & $-.20^{* *}$ & $-.06^{* * *}$ & $-.12^{\text {*** }}$ & -.04 & $-.05^{*}$ & $-.12^{* * *}$ & $-.06^{*}$ & $50.9 \%$ & \\
\hline 12. Marital status: married & .00 & $-.09^{* *}$ & $-.08^{* *}$ & $-.12^{* *}$ & $-.06^{* *}$ & $-.09^{* *}$ & -.05 & -.03 & $-.12^{* *}$ & $65.3 \%$ & \\
\hline 13. Marital status: divorced/ separated/ widow & -.02 & .04 & $.05^{*}$ & $.10^{* *}$ & .04 & .03 & .01 & -.01 & .04 & $22.9 \%$ & \\
\hline 14. Marital status: never married & .02 & $.09^{* * *}$ & $.04^{*}$ & $.04^{*}$ & $.05^{*}$ & $.09^{* *}$ & $.06^{*}$ & .05 & $.12^{* *}$ & $11.8 \%$ & \\
\hline 15. Education (level) & $.22^{* * *}$ & .03 & .04 & -.04 & $-.06^{* *}$ & .02 & -.04 & -.04 & .01 & $2.13(1.2)$ & $1-4$ \\
\hline 16. Ethnicity: White & .04 & .00 & .02 & -.03 & .01 & -.03 & -.03 & .04 & $-.06^{*}$ & $93.0 \%$ & \\
\hline 17. Ethnicity: Black & .00 & .01 & -.01 & $.06^{* *}$ & -.01 & .04 & .01 & -.03 & .04 & $2.2 \%$ & \\
\hline 18. Ethnicity: Aboriginal & -.03 & .04 & -.01 & .01 & .03 & .03 & .03 & -.02 & .01 & $.4 \%$ & \\
\hline 19. Ethnicity: Hispanic & -.04 & -.02 & -.02 & -.02 & -.03 & -.02 & .05 & .00 & $.08^{* *}$ & $.9 \%$ & \\
\hline 20. Ethnicity: South Asian & .03 & -.01 & .01 & -.01 & -.01 & -.02 & -.01 & -.02 & -.03 & $.2 \%$ & \\
\hline 21. Ethnicity: Asian & $-.05^{*}$ & -.02 & -.03 & -.01 & .01 & .01 & -.02 & -.02 & -.01 & $.3 \%$ & \\
\hline 22. Number of physical comorbidities & $.05^{*}$ & $.21^{* * *}$ & $.06^{* * *}$ & $.22^{* * *}$ & $.22^{* *}$ & $.14^{* *}$ & $.20^{* *}$ & $.22^{* * *}$ & $.14^{* *}$ & $1.79(1.5)$ & $0-8$ \\
\hline 23. Diabetes duration (years) & -.04 & .00 & .04 & -.03 & -.03 & -.03 & $.07^{* *}$ & .05 & -.02 & $4.59(3.1)$ & $0-10$ \\
\hline 24. Diabetes complications & .00 & $.16^{* * *}$ & $.05^{*}$ & $.17^{* *}$ & $.16^{* *}$ & $.21^{* * *}$ & $.17^{* *}$ & $.13^{* * *}$ & $.15^{* *}$ & $1.11(1.2)$ & $0-6$ \\
\hline
\end{tabular}

Note. Mental health conditions, sex, marital status, and ethnicity coded such that $0=$ no, $1=$ yes to indicated level; $\%$ indicates percentage of entire sample.

${ }^{*} p<.05 . \quad{ }^{* *} p<.01$ 
Table 2

Cross-Sectional and Prospective Associations Between Coping Strategies (Predictor) and Major Depression Syndrome, Elevated Anxiety Symptoms, and Moderate/Severe Diabetes-Related Distress (Outcomes)

\begin{tabular}{|c|c|c|c|c|c|c|c|c|}
\hline \multirow[t]{2}{*}{ Variable } & \multicolumn{4}{|c|}{ Cross-sectional analysis } & \multicolumn{4}{|c|}{ Prospective analysis } \\
\hline & $B$ & OR & $95 \% \mathrm{CI}$ & $p$ & $B$ & OR & $95 \% \mathrm{CI}$ & $p$ \\
\hline Major depression syndrome & \multicolumn{4}{|c|}{$(n=1,742)$} & \multicolumn{4}{|c|}{$(n=1,044)$} \\
\hline Task-oriented & -.42 & .66 & $.44, \quad .98$ & .041 & .22 & 1.24 & $.75,2.06$ & .401 \\
\hline Emotion-oriented & 1.84 & 6.31 & $4.36,9.13$ & $<.001$ & .92 & 2.51 & $1.57,4.00$ & $<.001$ \\
\hline Avoidance-oriented & -.27 & .76 & $.49, \quad 1.18$ & .224 & -.33 & .72 & $.42,1.26$ & .250 \\
\hline Elevated anxiety symptoms & \multicolumn{4}{|c|}{$(n=1,739)$} & \multicolumn{4}{|c|}{$(n=763)$} \\
\hline Task-oriented & -.45 & .64 & $.45, \quad .90$ & .010 & .14 & 1.15 & $.64,2.07$ & .651 \\
\hline Emotion-oriented & 2.06 & 7.84 & $5.63,10.93$ & $<.001$ & .87 & 2.38 & $1.19,4.73$ & .014 \\
\hline Avoidance-oriented & -.17 & .85 & $.59, \quad 1.22$ & .371 & -.11 & .90 & $.46,1.76$ & .749 \\
\hline Moderate/severe diabetes-related distress & \multicolumn{4}{|c|}{$(n=1,747)$} & \multicolumn{4}{|c|}{$(n=951)$} \\
\hline Task-oriented & -.21 & .82 & $.67, \quad .98$ & .029 & -.21 & .81 & $.62,1.07$ & .143 \\
\hline Emotion-oriented & 1.07 & 2.93 & $2.40, \quad 3.57$ & $<.001$ & .79 & 2.21 & $1.63,2.99$ & $<.001$ \\
\hline Avoidance-oriented & .18 & 1.20 & $.97, \quad 1.49$ & .101 & .03 & 1.03 & $.76,1.39$ & .871 \\
\hline
\end{tabular}

Note. All analyses reflect binary logistic regression models adjusted for covariates (age, number of other chronic conditions, diabetes duration, diabetes complication index, sex, marital status, education, and ethnicity), which were entered into the first step of regression models. Major depression syndrome: prospective = participants neither minor nor major depression syndrome at baseline (PHQ-9); predicting major depression syndrome at follow-up 1 and/or follow-up 2; 45 cases of major depression syndrome at follow up. Elevated anxiety symptoms: prospective = participants with "no anxiety" (GAD-7 < 5) at baseline; predicting elevated anxiety symptoms at follow-up 1 and/or follow-up 2; 26 cases of elevated anxiety symptoms at follow up. Moderate/severe diabetes-related distress: prospective $=$ participants with "little or no distress" (Diabetes Distress Scale $<2.0$ ) at baseline; predicting moderate/ high diabetes-related distress at follow-up 1 and/or follow-up 2; 150 cases of moderate/major diabetes-related distress at follow-up.

\section{What Are the Associations Between Coping Strategies and Major Depression Syndrome?}

Cross-sectional analysis. The first step of the model, which contained the covariates, was statistically significant, $\chi^{2}(15)=$ 111.02, $p<.001$, Nagelkerke $R^{2}=.14$. The second step of the model, which included the coping strategies, was also significant, $\chi^{2}(3)=218.76, p<.001$, Nagelkerke $R^{2}=.39$. Task-oriented coping was negatively associated with the likelihood of major depression syndrome, and emotion-oriented coping was positively associated with the likelihood of major depression syndrome. Avoidance-oriented coping showed no association (see Table 2). ${ }^{1}$

Prospective analysis. The first step of the model, which contained the covariates, was statistically significant, $\chi^{2}(15)=56.93$, $p<.001$, Nagelkerke $R^{2}=.18$. The second step of the model, which included the coping strategies, was also significant, $\chi^{2}(3)=$ $124.32, p=.001$, Nagelkerke $R^{2}=.38$. Emotion-oriented coping was positively associated with the likelihood of developing major depression syndrome during follow-up. Neither task-oriented nor avoidant-oriented coping was associated with the likelihood of developing major depression syndrome (see Table 2 and Footnote 1).

\section{What Are the Associations Between Coping Strategies and Elevated Anxiety Symptoms?}

Cross-sectional analysis. The first step of the model was statistically significant, $\chi^{2}(15)=111.02, p<.001$, Nagelkerke $R^{2}=.14$, as was the second step, $\chi^{2}(3)=218.76, p<.001$, Nagelkerke $R^{2}=.39$. Task-oriented coping was negatively associated with the likelihood of elevated anxiety symptoms, and emotion-oriented coping was positively associated with the likelihood of elevated anxiety symptoms Avoidance-oriented showed no association (see Table 2 and Footnote 1).

Prospective analysis. The first step of the model was not statistically significant, $\chi^{2}(15)=15.61, p=.41$, Nagelkerke
$R^{2}=.079$, though the second step of the model was, $\chi^{2}(3)=$ 7.97, $p=.047$ Nagelkerke $R^{2}=.12$. Emotion-oriented coping was positively associated with the likelihood of developing elevated anxiety symptoms during follow-up. Neither taskoriented nor avoidant-oriented coping was associated with the likelihood of developing elevated anxiety symptoms (see Table 2 and Footnote 1).

\section{What Are the Associations Between Coping Strategies and Moderate or Severe Diabetes-Related Distress?}

Cross-sectional analysis. The first step of the model was statistically significant, $\chi^{2}(15)=126.56, p<.001$, Nagelkerke $R^{2}=.11$, as was the second step, $\chi^{2}(3)=152.35, p<.001$, Nagelkerke $R^{2}=.23$. Task-oriented coping was negatively associated with the likelihood of moderate or severe diabetes-related distress, and emotion-oriented coping was positively associated with the likelihood of moderate or severe diabetes-related distress. Avoidance-oriented coping showed no association (see Table 2 and Footnote 1).

Prospective analysis. The first step of the model was not statistically significant, $\chi^{2}(15)=54.22, p<.001$, Nagelkerke $R^{2}=.095$, though the second step was, $\chi^{2}(3)=28.44, p<.001$, Nagelkerke $R^{2}=.14$. Emotion-oriented coping was positively associated with the likelihood of developing moderate or severe diabetes-related distress during follow-up. Neither task-oriented nor avoidant-oriented coping was associated with the likelihood of developing moderate or severe diabetes-related distress (see Table 2 and Footnote 1).

\footnotetext{
${ }^{1}$ Scoring measures of mental health continuously produced a consistent pattern of results (See Table 1 in the online supplemental materials).
} 


\section{Discussion}

Research on the associations between coping strategies and depression, anxiety, and diabetes-related distress in individuals with diabetes has been limited to cross-sectional data and has largely focused on individuals with type 1 diabetes. The present analyses expand this literature by providing evidence of the prospective relations between coping strategies and mental health outcomes in a large community sample of individuals with type 2 diabetes. Cross-sectionally, task-oriented coping was negatively associated with the likelihood of major depression syndrome, elevated anxiety symptoms, and moderate to severe diabetesrelated distress, whereas emotion-oriented coping was positively associated with the likelihood of these conditions. This pattern of results did not hold prospectively. Task-oriented coping was not protective against the development of these mental health conditions during a 2-year follow-up. Only emotion-oriented coping was associated with the development of these conditions, such that higher emotion-oriented coping scores were associated with an increased likelihood of developing major depression syndrome, elevated anxiety symptoms, and moderate or severe diabetesrelated distress.

The cross-sectional results are consistent with several prior studies (Karlsen et al., 2004; Smári \& Valtýsdóttir, 1997; Tuncay et al., 2008). Type 2 diabetes is distinct from other chronic conditions because many of the factors that influence its severity are controllable; the condition can be avoided or well managed with adherence to a healthy lifestyle and prescribed medication (Magkos, Yannakoulia, Chan, \& Mantzoros, 2009). Therefore, the cross-sectional pattern of results is consistent with work in the broader coping literature, which finds that task-oriented coping is usually adaptive, but emotion-oriented is not, if a stressor is controllable (Folkman, 1984). Cross-sectional results are also consistent with limited research that found no associations between avoidance-oriented coping and mental health outcomes in individuals with diabetes (Smári \& Valtýsdóttir, 1997).

Results from the prospective analyses differed from crosssectional results thereby underscoring the importance of complementing cross-sectional research with prospective studies. Taskoriented coping was associated with an increased likelihood of major depression syndrome, elevated anxiety symptoms and moderate to severe diabetes-related distress when mental health conditions were measured concurrently. However, task-oriented coping was not associated with the development of these conditions among individuals who did not meet the criteria for these conditions at baseline. Though further research is needed to explain why the observed cross-sectional association did not hold prospectively, this pattern of results suggests that mental health conditions may influence task-oriented coping, rather than task-oriented coping influencing mental health; depression, anxiety, and distress may hamper the ability of individuals with type 2 diabetes to employ task-oriented coping strategies. Indeed, hallmark symptoms of depression, such as behavioral inactivation and lack of motivation, impair one's ability to carry out a host of tasks, presumably including those involved in the management of stressors. Unfortunately, the single measurement of coping strategies at baseline precluded us from exploring this possibility. Therefore, future research in this area should measure coping strategies and mental health at several time points. Such research further affords the possibility of exploring how changes in coping strategies over time are associated with changes in mental health. Broadly, the possible bidirectional associations between coping strategies and mental health among people with type 2 diabetes are not wellexplored and may be promising avenues for future research.

Results from the prospective analyses further suggest that emotion-oriented coping plays a singular role in the onset of major depression syndrome, elevated anxiety symptoms, and moderate or severe diabetes-related distress. This is consistent with speculation that mental health outcomes are more sensitive to coping strategies that directly influence affective states (Duangdao \& Roesch, 2008). In interpreting the prospective associations between emotionoriented coping and mental health, it is important to consider the items on the emotion-oriented coping subscale of the CISS-21 (e.g., feel anxious about not being able to cope). The items query the extent to which negative affect is experienced when confronted with a stressor because the measure assumes that the presence of negative affect indicates that strategies to manage negative affect are not employed or successful.

The transactional model of stress and coping (Lazarus \& Folkman, 1987) posits that primary appraisal, which is the subjective evaluation of how threatening or challenging a stressor is, precedes secondary appraisal, which is the subjective evaluation of coping options and resources available to manage the stressor. The present study pertains primarily to secondary appraisal. Therefore, a more complete understanding of the role of coping in the onset of mental health conditions in individuals with type 2 diabetes can be garnered by investigating primary appraisal in future research. The present study also examined the coping strategies that individuals with type 2 diabetes usually employ when confronted with a stressor. Although individuals tend to have preferred coping strategies (Moos \& Holahan, 2003), people may also have domainspecific coping strategies (Stone, Greenberg, Kennedy-Moore, \& Newman, 1991). Diabetes can place a host of unique burdens on individuals, such as insulin injections and the threat of severe complications, which may elicit the development of specific coping strategies. Therefore, future work should seek to replicate these results with measures of diabetes-specific coping strategies.

\section{Strengths and Limitations}

To our knowledge, this study is the first to examine prospective associations between coping strategies and the development of major depression syndrome, elevated anxiety symptoms and moderate to severe diabetes-related distress in individuals with type 2 diabetes. This study has several strengths, including the use of a large community sample. The large sample size afforded us the unique opportunity to examine the associations between coping strategies and incident major depression syndrome, elevated anxiety symptoms, and moderate to severe diabetes-related distress. By excluding individuals who had subthreshold levels of depressive symptoms, anxiety symptoms, and diabetes-related distress at baseline, we were able to conduct a rigorous examination of the role of coping in the onset of these conditions. Conducting both cross-sectional and prospective analyses with the sample permitted direct comparison of the results of these analyses.

Despite its strengths, this study had several limitations. First, the study measured coping strategies with the CISS-21. This measure is widely used and has been validated (Endler \& Parker, 1994); 
however, its emotion-oriented subscale has been criticized for combining approach (e.g., I get upset) and avoidance (e.g., "I try not to think about it") strategies into a single subscale. Therefore, emotion-oriented items may be confounded with distress (e.g., I get upset), which may inflate the correlation between emotionoriented coping and conditions that are rooted in distress, such as depression, anxiety and diabetes-related distress (Stanton, DanoffBurg, Cameron \& Ellis, 1994; Folkman \& Moskowitz, 2004). Unfortunately, this criticism applies to a number of other measures of coping strategies, so as measures of coping that circumvent these issues become available, it will be important to incorporate them into longitudinal studies of coping and mental health outcomes in populations with type 2 diabetes. Second, the measures of depression and anxiety that were used are screening tools; they are not diagnostic assessment tools. Future work should seek to replicate these findings with clinical assessments of depression and anxiety. Third, the eligibility criteria for participation produced a fairly homogeneous sample, which limits generalizability. Although this proffers some benefits, the sample did not contain individuals with a long history of diabetes. Moreover, because individuals who reported taking insulin injections at baseline were not eligible to participate, it is likely that our sample was relatively healthy. By examining mental health during a 2-year follow-up, the associations between coping and mental health were studied during a period that participants were likely relatively healthy. Because type 2 diabetes is a progressive disease, we would expect mental health to decline as disease severity and burden increase over time. Therefore, the observed pattern of results may change as longer follow-up periods are examined. The observed pattern of results may differ between individuals who have been diagnosed with diabetes for many years relative to those who are newly diagnosed. Unfortunately, the restricted range of diabetes duration in the present study (10 years or less) was a limitation that did not permit a strong test of this possibility. ${ }^{2}$ Finally, diabetes diagnosis was self-reported, which introduces the possibility of bias.

\section{Implications for Intervention Development}

Because individuals with type 2 diabetes are at increased risk of mental health conditions, such as depression, anxiety, and distress, and these conditions are associated with factors that may intensify diabetes severity (de Groot, et al., 2001), there is growing interest in developing interventions to protect the mental health of individuals with type 2 diabetes. Coping is a suitable intervention target to consider in this context because coping strategies are modifiable with cognitive-behavioral interventions (Folkman \& Moskowitz, 2004). In fact, the modification and development of coping strategies is often included in the treatment of depression in people with diabetes (Duangdao \& Roesch, 2008; Grey \& Berry, 2004). Though many interventions focus on bolstering taskoriented coping by teaching skills and boosting self-efficacy (Grey \& Berry, 2004), the results of the current study suggest that it may be most beneficial to target emotion-oriented coping. Specifically, it may be useful to target strategies that blunt the experience of negative affect when confronted with a stressor. Interventionists are encouraged to distinguish between and target specific types of coping strategies because doing so may improve intervention effectiveness and efficiency.
Multifaceted interventions (i.e., targeting clinical, behavioral and psychosocial outcomes) that focus on prevention are becoming increasingly popular in the context of diabetes care (Piatt et al., 2010). To balance the effectiveness of these interventions against scarce resources, it is useful to design intervention components that will influence several outcomes whenever possible. From this perspective, emotion-oriented coping may be a suitable construct to target because (a) it was associated with each of the three mental health conditions examined, and (b) depression, anxiety, and diabetes distress are among the most common psychological conditions among people with type 2 diabetes. Results of the present study suggest that targeting emotion-oriented coping may curb the development of all of these conditions and thus may eliminate the need for isolated interventions targeting each of depression, anxiety, and diabetes-related distress.

${ }^{2}$ All analyses were repeated using diabetes duration as a moderator, rather than as a covariate. No interaction terms were significant.

\section{References}

Alberti, K. G. M. M., \& Zimmet, P. Z. (1998). Definition, diagnosis and classification of diabetes mellitus and its complications. Pt. 1: Diagnosis and classification of diabetes mellitus provisional report of a WHO consultation. Diabetic Medicine, 15, 539-553. http://dx.doi.org/ 10.1002/(SICI)1096-9136(199807)15:7<539::AID-DIA668>3.0.CO;2-S

Cole, M. G., \& Dendukuri, N. (2003). Risk factors for depression among elderly community subjects: A systematic review and meta-analysis. Risk, 160, 1147-1156. http://dx.doi.org/10.1176/appi.ajp.160.6.1147

de Groot, M., Anderson, R., Freedland, K. E., Clouse, R. E., \& Lustman, P. J. (2001). Association of depression and diabetes complications: A meta-analysis. Psychosomatic Medicine, 63, 619-630. http://dx.doi.org/ 10.1097/00006842-200107000-00015

D’Eramo Melkus, G., Chyun, D., Vorderstrasse, A., Newlin, K., Jefferson, V., \& Langerman, S. (2010). The effect of a diabetes education, coping skills training, and care intervention on physiological and psychosocial outcomes in black women with Type 2 diabetes. Biological Research for Nursing, 12, 7-19. http://dx.doi.org/10.1177/1099800410369825

Duangdao, K. M., \& Roesch, S. C. (2008). Coping with diabetes in adulthood: A meta-analysis. Journal of Behavioral Medicine, 31, 291300. http://dx.doi.org/10.1007/s10865-008-9155-6

Eaton, W. W., Hall, A. L., Macdonald, R., \& McKibben, J. (2007). Case identification in psychiatric epidemiology: A review. International Review of Psychiatry, 19, 497-507. http://dx.doi.org/10.1080/ 09540260701564906

Endler, N. S., \& Parker, J. D. (1990). Multidimensional assessment of coping: A critical evaluation. Journal of Personality and Social Psychology, 58, 844-854. http://dx.doi.org/10.1037/0022-3514.58.5.844

Endler, N. S., \& Parker, J. D. (1994). Assessment of multidimensional coping: Task, emotion, and avoidance strategies. Psychological Assessment, 6, 50-60. http://dx.doi.org/10.1037/1040-3590.6.1.50

Fincke, B. G., Clark, J. A., Linzer, M., Spiro, A., III Miller, D. R., Lee, A., \& Kazis, L. E. (2005). Assessment of long-term complications due to Type 2 diabetes using patient self-report: The diabetes complications index. The Journal of Ambulatory Care Management, 28, 262-273. http://dx.doi.org/10.1097/00004479-200507000-00010

Fisher, L., Gonzalez, J. S., \& Polonsky, W. H. (2014). The confusing tale of depression and distress in patients with diabetes: A call for greater clarity and precision. Diabetic Medicine, 31, 764-772. http://dx.doi.org/ 10.1111/dme. 12428

Fisher, L., Hessler, D. M., Polonsky, W. H., \& Mullan, J. (2012). When is diabetes distress clinically meaningful? Establishing cut points for the 
Diabetes Distress Scale. Diabetes Care, 35, 259-264. http://dx.doi.org/ $10.2337 / \mathrm{dc} 11-1572$

Fisher, L., Mullan, J. T., Skaff, M. M., Glasgow, R. E., Arean, P., \& Hessler, D. (2009). Predicting diabetes distress in patients with Type 2 diabetes: A longitudinal study. Diabetic Medicine, 26, 622-627. http:// dx.doi.org/10.1111/j.1464-5491.2009.02730.x

Fisher, L., Skaff, M. M., Mullan, J. T., Arean, P., Glasgow, R., \& Masharani, U. (2008). A longitudinal study of affective and anxiety disorders, depressive affect and diabetes distress in adults with Type 2 diabetes. Diabetic Medicine, 25, 1096-1101. http://dx.doi.org/10.1111/j.14645491.2008.02533.x

Folkman, S. (1984). Personal control and stress and coping processes: A theoretical analysis. Journal of Personality and Social Psychology, 46, 839-852. http://dx.doi.org/10.1037/0022-3514.46.4.839

Folkman, S., \& Lazarus, R. S. (1980). An analysis of coping in a middleaged community sample. Journal of Health and Social Behavior, 21, 219-239. http://dx.doi.org/10.2307/2136617

Folkman, S., \& Moskowitz, J. T. (2004). Coping: Pitfalls and promise. Annual Review of Psychology, 55, 745-774. http://dx.doi.org/10.1146/ annurev.psych.55.090902.141456

Gåfvels, C., \& Wändell, P. E. (2006). Coping strategies in men and women with type 2 diabetes in Swedish primary care. Diabetes Research and Clinical Practice, 71, 280-289. http://dx.doi.org/10.1016/j.diabres.2005 .07 .001

Gonzalez, J. S., Fisher, L., \& Polonsky, W. H. (2011). Depression in diabetes: Have we been missing something important? Diabetes Care, 34, 236-239. http://dx.doi.org/10.2337/dc10-1970

Grey, M., \& Berry, D. (2004). Coping skills training and problem solving in diabetes. Current Diabetes Reports, 4, 126-131. http://dx.doi.org/ 10.1007/s11892-004-0068-7

Hendriks, S. M., Spijker, J., Licht, C. M., Beekman, A. T., Hardeveld, F., de Graaf, R., . . Penninx, B. W. (2014). Disability in anxiety disorders. Journal of Affective Disorders, 166, 227-233. http://dx.doi.org/10.1016/ j.jad.2014.05.006

Horwath, E., Johnson, J., Klerman, G. L., \& Weissman, M. M. (1992). Depressive symptoms as relative and attributable risk factors for firstonset major depression. Archives of General Psychiatry, 49, 817-823. http://dx.doi.org/10.1001/archpsyc.1992.01820100061011

Karlsen, B., Idsoe, T., Dirdal, I., Rokne Hanestad, B., \& Bru, E. (2004). Effects of a group-based counselling programme on diabetes-related stress, coping, psychological well-being and metabolic control in adults with type 1 or type 2 diabetes. Patient Education and Counseling, 53, 299-308. http://dx.doi.org/10.1016/j.pec.2003.10.008

Karlsen, B., Oftedal, B., \& Bru, E. (2012). The relationship between clinical indicators, coping styles, perceived support and diabetes-related distress among adults with type 2 diabetes. Journal of Advanced Nursing, 68, 391-401. http://dx.doi.org/10.1111/j.1365-2648.2011.05751.x

Kroenke, K., Spitzer, R. L., \& Williams, J. B. (2001). The PHQ-9: Validity of a brief depression severity measure. Journal of General Internal Medicine, 16, 606-613. http://dx.doi.org/10.1046/j.1525-1497.2001 $.016009606 . x$

Kroese, F. M., Adriaanse, M. A., Vinkers, C. D., van de Schoot, R., \& de Ridder, D. T. (2013). The effectiveness of a proactive coping intervention targeting self-management in diabetes patients. Psychology \& Health, 29, 110-125. http://dx.doi.org/10.1080/08870446.2013.841911

Lazarus, R. S. (1993). Coping theory and research: Past, present, and future. Psychosomatic Medicine, 55, 234-247. http://dx.doi.org/ 10.1097/00006842-199305000-00002

Lazarus, R. S., \& Folkman, S. (1987). Transactional theory and research on emotions and coping. European Journal of Personality, 1, 141-169. http://dx.doi.org/10.1002/per.2410010304

Lecrubier, Y. (2001). The burden of depression and anxiety in general medicine. The Journal of Clinical Psychiatry, 62(Suppl. 8), 4-9.
Lorant, V., Croux, C., Weich, S., Deliège, D., Mackenbach, J., \& Ansseau, M. (2007). Depression and socio-economic risk factors: 7-year longitudinal population study. The British Journal of Psychiatry, 190, 293-298. http://dx.doi.org/10.1192/bjp.bp.105.020040

Macrodimitris, S. D., \& Endler, N. S. (2001). Coping, control, and adjustment in Type 2 diabetes. Health Psychology, 20, 208-216. http://dx.doi org/10.1037/0278-6133.20.3.208

Magkos, F., Yannakoulia, M., Chan, J. L., \& Mantzoros, C. S. (2009). Management of the metabolic syndrome and type 2 diabetes through lifestyle modification. Annual Review of Nutrition, 29, 223-256. http:// dx.doi.org/10.1146/annurev-nutr-080508-141200

Mezuk, B., Eaton, W. W., Albrecht, S., \& Golden, S. H. (2008). Depression and Type 2 diabetes over the lifespan: A meta-analysis. Diabetes Care, 31, 2383-2390. http://dx.doi.org/10.2337/dc08-0985

Moos, R. H., \& Holahan, C. J. (2003). Dispositional and contextual perspectives on coping: Toward an integrative framework. Journal of Clinical Psychology, 59, 1387-1403. http://dx.doi.org/10.1002/jclp .10229

Moos, R. H., \& Moos, B. S. (1988). Coping responses inventory manual. Palo Alto, CA; Center for Health Care Evaluation, Department of Veteran Affairs and Stanford University Medical Centers.

Piatt, G. A., Anderson, R. M., Brooks, M. M., Songer, T., Siminerio, L. M., Korytkowski, M. M., \& Zgibor, J. C. (2010). 3-year follow-up of clinical and behavioral improvements following a multifaceted diabetes care intervention: Results of a randomized controlled trial. The Diabetes Educator, 36, 301-309. http://dx.doi.org/10.1177/0145721710361388

Polonsky, W. H., Fisher, L., Earles, J., Dudl, R. J., Lees, J., Mullan, J., \& Jackson, R. A. (2005). Assessing psychosocial distress in diabetes: Development of the diabetes distress scale. Diabetes Care, 28, 626-631. http://dx.doi.org/10.2337/diacare.28.3.626

Shah, B. M., Gupchup, G. V., Borrego, M. E., Raisch, D. W., \& Knapp K. K. (2012). Depressive symptoms in patients with Type 2 diabetes mellitus: Do stress and coping matter? Stress and Health, 28, 111-122. http://dx.doi.org/10.1002/smi.1410

Smári, J., \& Valtýsdóttir, H. (1997). Dispositional coping, psychological distress, and disease-control in diabetes. Personality and Individual Differences, 22, 151-156. http://dx.doi.org/10.1016/S0191-8869 (96)00199-7

Smith, K. J., Béland, M., Clyde, M., Gariépy, G., Pagé, V., Badawi, G., . . Schmitz, N. (2013). Association of diabetes with anxiety: A systematic review and meta-analysis. Journal of Psychosomatic Research, 74, 8999. http://dx.doi.org/10.1016/j.jpsychores.2012.11.013

Smith, K. J., Gariépy, G., Pedneault, M., Beland, M., Clyde, M., \& Schmitz, N. (2013). Exploring the association of psychological status with self-rated diabetes control: Results from the Montreal evaluation of diabetes treatment study. Psychosomatics, 54, 35-43. http://dx.doi.org/ 10.1016/j.psym.2012.08.002

Smith, K. J., \& Schmitz, N. (2014). Association of depression and anxiety symptoms with functional disability and disability days in a community sample with Type 2 diabetes. Psychosomatics, 55, 659-667. http://dx .doi.org/10.1016/j.psym.2014.05.015

Spitzer, R. L., Kroenke, K., Williams, J. B., \& Löwe, B. (2006). A brief measure for assessing generalized anxiety disorder: The GAD-7. Archives of Internal Medicine, 166, 1092-1097. http://dx.doi.org/10.1001/ archinte.166.10.1092

Stanton, A. L., Danoff-Burg, S., Cameron, C. L., \& Ellis, A. P. (1994). Coping through emotional approach: Problems of conceptualization and confounding. Journal of Personality and Social Psychology, 66, 350362. http://dx.doi.org/10.1037/0022-3514.66.2.350

Stanton, A. L., Revenson, T. A., \& Tennen, H. (2007). Health psychology: Psychological adjustment to chronic disease. Annual Review of Psychology, 58, 565-592. http://dx.doi.org/10.1146/annurev.psych.58.110405 .085615 
Stone, A. A., Greenberg, M. A., Kennedy-Moore, E., \& Newman, M. G. (1991). Self-report, situation-specific coping questionnaires: What are they measuring? Journal of Personality and Social Psychology, 61, 648-658. http://dx.doi.org/10.1037/0022-3514.61.4.648

Tuncay, T., Musabak, I., Gok, D. E., \& Kutlu, M. (2008). The relationship between anxiety, coping strategies and characteristics of patients with diabetes. Health and Quality of Life Outcomes, 6, 79. http://dx.doi.org/ 10.1186/1477-7525-6-79

Wells, K. B., Burnam, M. A., Rogers, W., Hays, R., \& Camp, P. (1992). The course of depression in adult outpatients. Results from the Medical
Outcomes Study. Archives of General Psychiatry, 49, 788-794. http:// dx.doi.org/10.1001/archpsyc.1992.01820100032007

Received October 17, 2014

Revision received May 5, 2015

Accepted June 1, 2015 